\title{
Isolated Photons at CMS
}

\author{
Rong-Shyang Lu*† \\ National Taiwan University \\ E-mail: Rong-Shyang.Lu@cern.ch
}

We present the preliminary results on commissioning of photon reconstruction and identification toward the isolated photon production in the CMS experiment using $74 \mathrm{nb}^{-1}$ data collected in proton-proton collisions at $\sqrt{s}=7 \mathrm{TeV}$. In order to identify isolated photons, the contribution of background from jets and hadron decays, such as $\pi^{0}$ decays to two photons, are estimated with several variables, including the shower shape measured in the electromagnetic calorimeter (ECAL), the isolation measured in tracker and calorimeter, and the ratio of momentum measured in the tracker to the energy measured in the ECAL for converted photons. The results present the isolated photon production as expected from Monte-Carlo.

35th International Conference of High Energy Physics - ICHEP2010,

July 22-28, 2010

Paris France

\footnotetext{
*Speaker.

$\dagger$ on behalf of CMS Collaboration
} 


\section{Introduction}

The measurement of the isolated photon spectrum in pp collisions provides a precision test of perturbative quantum chromodynamics as well as information on the constituents of hadronic matter. In addition, this measurement plays a crucial role in the context of Higgs and new physics searches with photons as it allows us to understand one of the main standard model backgrounds. In high energy proton-proton collisions, prompt photons are produced either directly in the hard scattering or collinear fragmentation of a parton producing large transverse momentum. Prompt photons are the signal of the measurement, with respect to the background from electromagnetic showers resulting from decays of neutral hadrons in jets such as $\pi^{0}$ and $\eta^{0}$ mesons.

\section{CMS experiment}

The CMS detector is a general-purpose detector built to explore the physics at the $\mathrm{TeV}$ scale [1]. The CMS electromagnetic calorimeter (ECAL) is composed of lead tungstate crystals and designed to have both excellent energy resolution and high granularity. The ECAL is organized into two pseudo-rapidity regions: the ECAL barrel (EB, $|\eta|<1.479)$ and the ECAL endcap (EE, $1.479<|\eta|<3.0$ ). In the endcap region, there is a lead-silicon-strip preshower detector (ES) consisting of two orthogonal silicon strip detectors with strip pitch of $1.9 \mathrm{~mm}$. One plane of the detector is at a depth of $2 X_{0}$ and the other is at $3 X_{0}$. The ECAL is surrounded by a brass/scintillator sampling hadron calorimeter (HCAL) with coverage up to $|\eta|<3.0$. The CMS tracking system is composed of several layers of silicon pixel and silicon strip detectors and covers the region of $|\eta|<2.5$. The tracker and both calorimeters are embedded inside a 3.8 Tesla solenoidal magnet. Data used for the analysis corresponds to $74 \mathrm{nb}^{-1}$ collected at $\sqrt{s}=7 \mathrm{TeV}$ of LHC collision energy.

\section{Supercluster and Photon}

An ECAL supercluster is a collection of sub-clusters extended in the $\phi$ direction in order to recover the energy spread due to material in front of ECAL and strong magnetic field, that causes conversion electrons to spread over large distance in $\phi$. The comparison of supercluster $\eta$ and raw $E_{T}$ distributions between data and Monte-Carlo is shown in Fig. 1. The supercluster has no energy corrections and the transverse energy is calculated using the nominal interaction point. Good agreement is found between data and Monte-Carlo. To avoid the gap between EB and EE, an $\mathrm{EB}(\mathrm{EE})$ supercluster is required to be in the region of $|\eta|<1.4442(1.566<|\eta|<2.5)$.

Photon objects are constructed from the superclusters by assigning the candidate momentum to the location of the primary vertex. The energy correction on superclusters are typically at the $1 \%$ level depends on the supercluster $E_{T}$ and $\eta$.

\section{Photon Identification}

Photon identification uses a set of selection criteria that have been defined based on MonteCarlo simulation. The photon identification aims to increase photon purity and maximizing background rejection while keeping efficiency flat as a function of $E_{T}$ and $\eta$. The photon identification uses the following characteristics. 


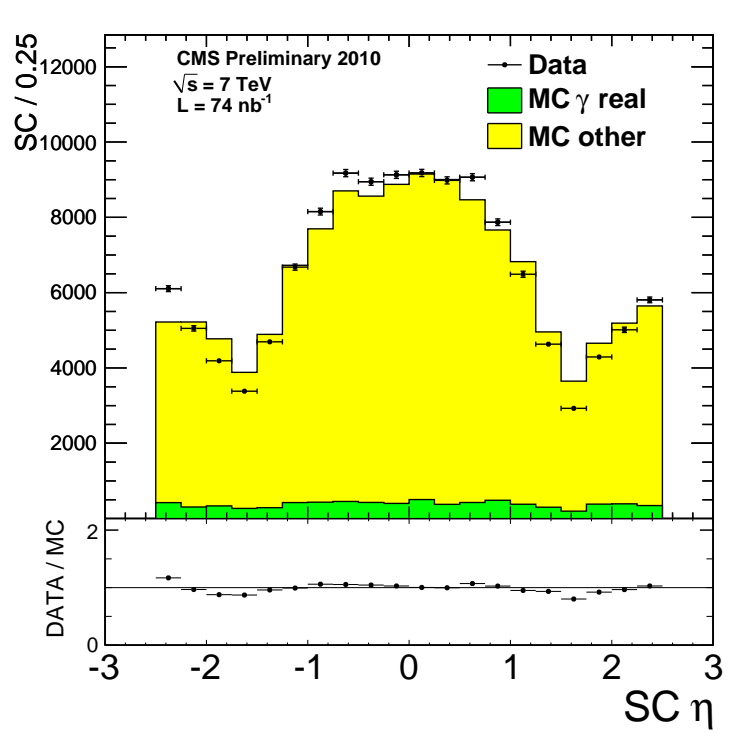

(a)

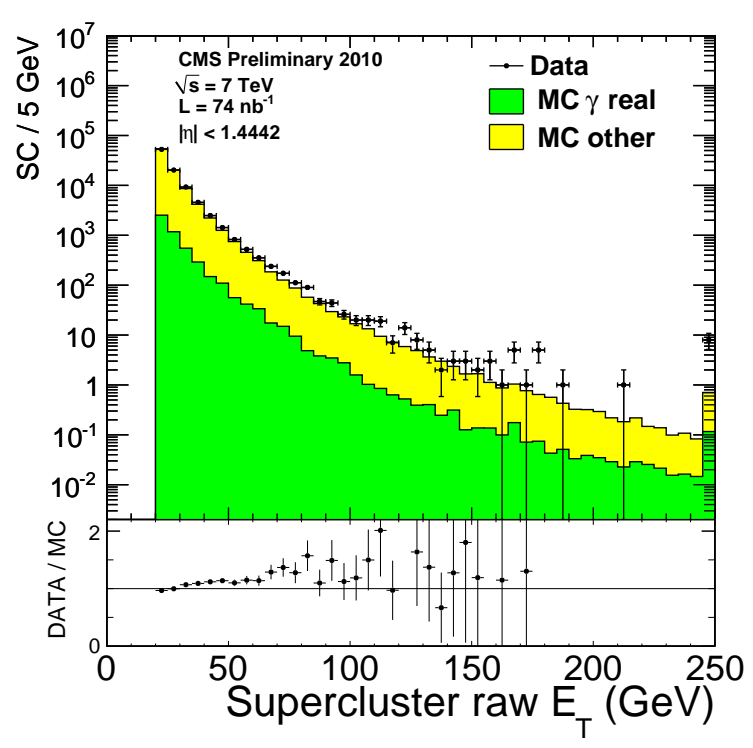

(b)

Figure 1: The supercluster candidates reconstructed in CMS ECAL with $\eta$ distribution (a) and Pt distribution of EB region (b). The Monte-Carlo distributions are normalized to the same area of data.

- The supercluster is required not to match pixel hits consistent with a track from the interaction region.

- ECAL isolation: the sum of ECAL $E_{T}$ around the photon candidate in an annular region of inner radius $\mathrm{R}=\sqrt{(\Delta \eta)^{2}+(\Delta \phi)^{2}}=0.06$ and outer radius 0.4 . A three-crystal wide strip along $\phi$ is excluded.

- HCAL isolation: the sum of HCAL $E_{T}$ around the photon candidate in an annular region of inner radius $\mathrm{R}=0.15$ and outer radius 0.4 .

- Tracker isolation: the scalar sum of $p_{T}$ of tracks consistent with the primary vertex in a hollow cone around the photon candidate.

- $\sigma_{i \eta i \eta}$ is the $\eta-\eta$ element of the $\eta-\phi$ covariance matrix, which provides another expression for the extent in $\eta$ of the supercluster. It is given by:

$$
\sigma_{i \eta i \eta}^{2}=\frac{\sum_{i}^{5 \times 5} w_{i}\left(i \eta_{i}-i \eta_{\text {seed }}\right)^{2}}{\sum_{i}^{5 \times 5} w_{i}}, w_{i}=\max \left(0,4.7+\ln \frac{E_{i}}{E_{5 \times 5}}\right),
$$

where $\mathrm{E}_{i}$ and $i \eta_{i}$ are the energy and $\eta$ index of the $\mathrm{i}^{\text {th }}$ crystal within the $5 \times 5$ cluster, $E_{5 \times 5}$ is the energy of the $5 \times 5$ cluster, and $i \eta_{\text {seed }}$ is the $\eta$ index of the seed crystal.

- $\mathrm{H} / \mathrm{E}$ is the ratio of energy sum of HCAL towers $(\mathrm{H})$ behind the photon candidate and within $R<0.15$ over the electromagnetic energy (E) of photon candidate.

The values of the cuts adopted for the photon identification are shown in Table 1. Two distributions, the combined ECAL+HCAL+Tracker isolation and $\sigma_{i \eta i \eta}$, are shown in Fig. 2 with different 
Table 1: Photon identification thresholds for the barrel and the endcap regions.

\begin{tabular}{|c|cccccc|}
\hline Variable & pixel seed & Tracker Iso & ECAL Iso & HCAL Iso & $\mathrm{H} / \mathrm{E}$ & $\sigma_{i \eta i \eta}$ \\
\hline $\begin{array}{c}\text { Barrel } \\
\text { Endcap }\end{array}$ & require none & \multirow{2}{*}{$2.0 \mathrm{GeV}$} & \multirow{2}{*}{$4.2 \mathrm{GeV}$} & $2.2 \mathrm{GeV}$ & 0.05 & 0.01 \\
0.03
\end{tabular}

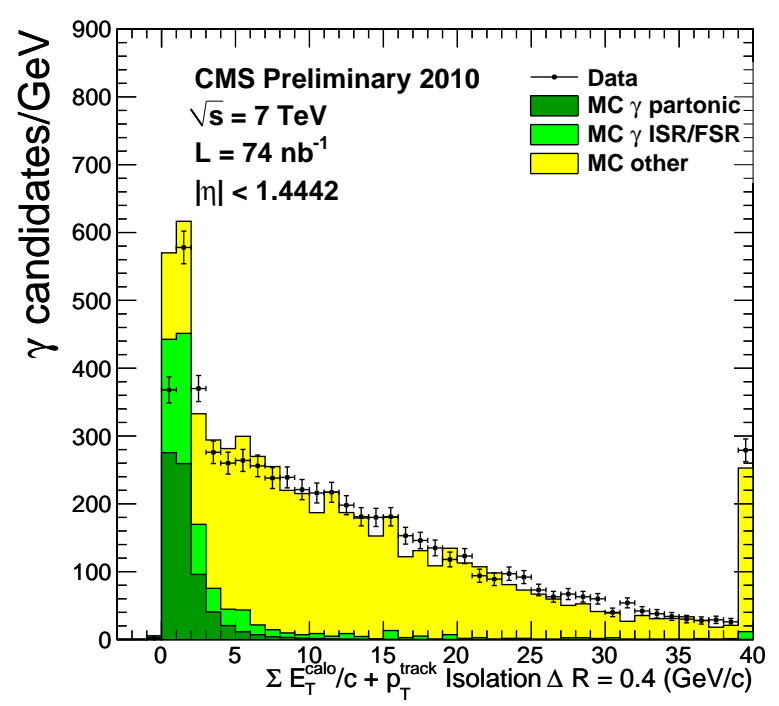

(a)

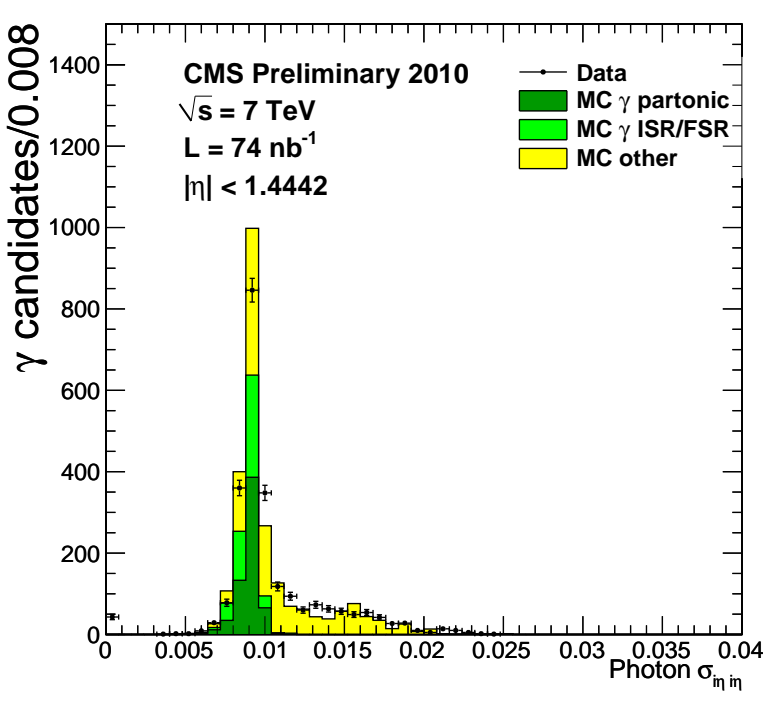

(b)

Figure 2: The combined (ECAL+HCAL+Tracker) isolation variable (a) and $\sigma_{i \eta i \eta}(\mathrm{b})$ with different contributions illustrated.

photon components including real photons coming from both hard interaction and ISR/FSR, as well as the background component coming from QCD are illustrated. With this selection, the Monte-Carlo predicts a purity of about $50 \%$ signal photons with $E_{T}>30 \mathrm{GeV}$. The efficiency of the selection on isolated photons in Monte Carlo is about $90 \%$ in the EB and $80 \%$ in EE.

The $E_{T}$ spectrum of isolated photon candidates, with above photon identification selections applied, are shown in Fig. 3(a), together with contributions from various photon source superimposed. Each of the contributions is estimated from Monte-Carlo simulation and normalized to the relative cross-section obtained from PYTHIA.

\section{Photon Conversions}

There are significant amount of photon conversions expected in CMS due to material in front of the ECAL which averages about $1 \mathrm{X}_{0}$. In addition to reconstructing the converted photon showers in ECAL superclusters, the conversion tracks can be reconstructed. The conversion tracks finder searches an outside-in track from the seed cluster of a supercluster. From the most inner hit of the track, an insider-out track are build. In both cases, the possible energy loss for electron tracks is considered. The possible combinations of oppositely charged tracks are selected and combined to form conversion track-pair candidates. Track-pairs coming from conversions are characterized 


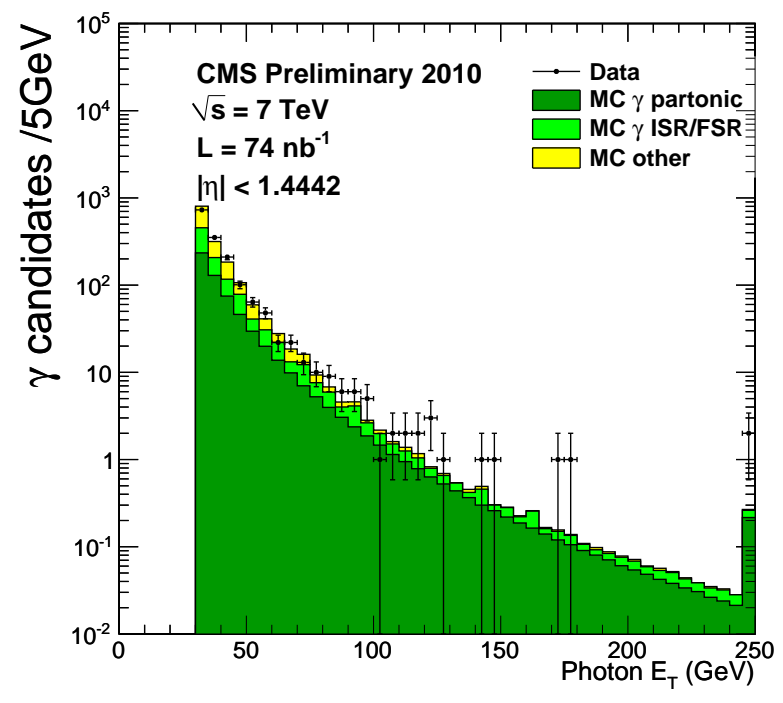

(a)

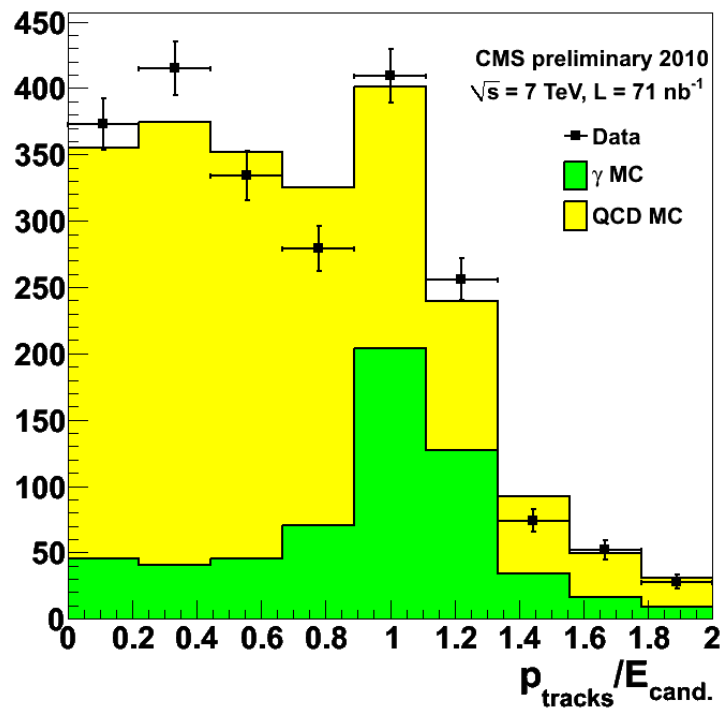

(b)

Figure 3: The $E_{T}$ distributions of isolated photon candidates in EB (a) and the track momentum $(P)$ over photon energy $E_{T}$ distributions (b) of conversion candidates with signal and background contribution also illustrated.

by a pair of oppositely charged tracks with small opening angle and an invariant mass consistent with zero. In addition, a good vertex fit probability is required. The distribution of the conversion track-pair momentum sum divided by supercluster energy (P/E), is shown in Fig. 3(b). The figure shows the isolated photon candidates with histrograms of the simulated signal and background superimposed. The figure shows $\mathrm{P} / \mathrm{E}$ for conversion track-pairs reconstructed from photons passing the photon identification cuts given Table 1 .

\section{Summary}

In summary, CMS has commissioned the reconstruction and identification of photon objects using early 2010 data. The performance of ECAL detector and reconstruction algorithm match our expectation. After applying a photon identification to suppress background, clear prompt photon signal can be seen in various kinematic distributions.

\section{References}

[1] CMS Collaboration, "The CMS experiment at the CERN LHC, JINST 3 (2008) S08004. doi:10.1088/1748-0221/3/08/S08004

[2] CMS Collaboration, Electromagnetic calorimeter commissioning and first results with $7 \mathrm{TeV}$ data, CMS Physics Analysis Summary EGM-10-002 (2010).

[3] CMS collaboration, "Photon reconstruction and identification at $\sqrt{s}=7 \mathrm{TeV}$ ", CMS Physics Analysis Summary EGM-10-005 (2010). 